\title{
Chemical Synapse
}

National Cancer Institute

\section{Source}

National Cancer Institute. Chemical Synapse. NCI Thesaurus. Code C32302.

A synapse in which an action potential causes the exocytosis of neurotransmitter from the presynaptic cell, which diffuses across the synaptic cleft and binds to ligand gated ion channels on the post synaptic cell. These ion channels then affect the resting potential of the post synaptic cell. 